\title{
Evaluation of a computer-based intervention to enhance metabolic monitoring in psychiatry inpatients treated with second-generation antipsychotics
}

\author{
M. T. DelMonte ${ }^{* 1}$ PharmD CPP BCPS, J. R. Bostwick † PharmD BCPS BCPP, J. D. Bess $\ddagger$ MD and G. W. Dalack $\$$ MD \\ ${ }^{*}$ Carolina Advanced Health, UNC Health Care, Department of Pharmacy Services, Chapel Hill, NC, †University of Michigan Health System, University \\ of Michigan College of Pharmacy, Ann Arbor, MI, and \$Department of Psychiatry, University of Michigan, Ann Arbor, MI, USA
}

Received 4 October 2011, Accepted 19 June 2012

Keywords: chronic mental illness, computerized physician order entry system, metabolic monitoring, metabolic syndrome, secondgeneration antipsychotics

\section{SUMMARY}

What is known and Objective: Second-generation antipsychotics (SGAs) play an important role in the pharmacologic management of various psychiatric conditions. Use of these medications has been associated with metabolic complications. Adherence to guideline-recommended monitoring is suboptimal. We evaluated the effect of a computerized physician order entry (CPOE) pop-up alert designed to improve rates of laboratory metabolic monitoring of patients treated with SGAs on a University Hospital inpatient psychiatry unit. Methods: A single-centre, retrospective chart review was performed in which patient demographics and SGA drug and laboratory data were extracted from the CPOE database. We assessed the number of orders for appropriate metabolic monitoring data for patients admitted within a 6-month period before or after the alert implementation.

Results and Discussion: Pre-alert $(n=171)$ and post-alert ( $n=157)$ groups were similar with respect to age, length of stay, sex, race and comorbidities. Following alert implementation, significant increases in monitoring both random $(92.4 \%$ vs. $100 \%)$ and fasting $(46.8 \%$ vs. $70 \%)$ glucose levels as well as random $(28.7 \%$ vs. $74.5 \%)$ and fasting (18.7\% vs. $59.9 \%)$ lipid panels (all $P \leq 0.001$ ) were observed. The number of patients with both a fasting glucose level and fasting lipid panel available for monitoring increased from $12.9 \%$ to $47.8 \%$ $(P<0.0001)$. Significantly more post-alert laboratory orders were submitted at the same time as the SGA drug order $(P<0.0001)$, suggesting that the alert itself had a direct influence on the ordering of metabolic monitoring labs.

What is new and Conclusions: Implementation and use of an electronic pop-up alert in an inpatient psychiatric unit significantly improved rates of ordering fasting blood glucose and lipid levels for inpatients treated with SGAs. Overall rates remain suboptimal, suggesting a need for additional strategies to further improve metabolic monitoring.

Correspondence: M. T. DelMonte, Carolina Advanced Health, UNC Health Care, Department of Pharmacy Services, Chapel Hill, NC, USA. Tel.: +1 734717 3449; fax: +1 919445 6011; e-mail: mdelmonte@ gmail.com

${ }^{1}$ Present address: Carolina Advanced Health, 6101 Quadrangle Drive, Suite 100, Chapel Hill, NC 27517, USA.

\section{WHAT IS KNOWN AND OBJECTIVE}

Second-generation antipsychotics (SGAs) play an important role in the pharmacologic management of patients with a variety of psychiatric conditions, including schizophrenia and bipolar disorder. ${ }^{1,2}$ Considered safer and better tolerated than their firstgeneration predecessors, SGAs produce fewer extrapyramidal symptoms and may be more effective in managing negative, cognitive and affective symptoms associated with many psychiatric disorders. ${ }^{1-3}$

Use of SGAs has been associated with metabolic complications, including obesity, diabetes and dyslipidaemia. ${ }^{2}$ The prevalence of metabolic syndrome in patients on SGA therapy can reach $30 \%$ or more, increasing the risk for developing coronary artery disease or stroke by 3 -fold..$^{3-5}$ Individuals with serious mental illnesses are already at an increased risk of mortality due in part to genetic risk factors, higher rates of smoking and inactivity, and lower socioeconomic status. ${ }^{2,6,7}$ Greater awareness of risks associated with SGA use is critical in this vulnerable population.

In February of 2004, a joint consensus statement addressed the evidence of metabolic risks and the need for ongoing monitoring in patients taking SGAs. ${ }^{2}$ The statement emphasized the importance of baseline and follow-up monitoring throughout the duration of treatment for all patients on SGAs, and a protocol was proposed recommending the frequency of monitoring various metabolic parameters. $^{2}$

Despite general acceptance of these guidelines, appropriate monitoring remains uncommon. ${ }^{8-11}$ One retrospective cohort including over 23000 patients on SGAs reported baseline lipid testing rates of $8.4 \%$ and glucose testing rates of $17.3 \%$. Rates rose only slightly after the 2004 consensus statement was issued (10.5\% and $21 \cdot 8 \%$, respectively). ${ }^{8}$ Other studies have shown similar results, with $<30 \%$ of patients receiving baseline glucose testing and $<10 \%$ receiving baseline lipid testing. ${ }^{9-11}$ In 2009, Morrato et al. ${ }^{10}$ concluded that publication of the 2004 ADA guidelines did not significantly increase metabolic testing in patients initiating SGA therapy.

Whereas a number of studies have confirmed the importance of monitoring metabolic parameters in patients on SGAs, ${ }^{1-4}$ little research exists on the development and implementation of an effective monitoring program within an inpatient setting. Several outpatient monitoring programs have been described. One focused on the collection of point of care measurements at a Universityaffiliated psychiatry clinic $^{12}$; the other assessed the implementation and use of a metabolic monitoring program incorporated into the electronic medical records of prisoners. ${ }^{4}$ Whereas both studies 
documented successful interventions, the authors of each indicated the need for further improvements in SGA-related metabolic monitoring within high-risk patient populations.

The recent implementation of a computerized physician order entry (CPOE) system at the University of Michigan Health System (UMHS) provided a unique opportunity to enhance metabolic monitoring in psychiatric patients treated with SGAs. Based on evidence demonstrating increased rates of medication-related laboratory testing through use of patient-specific electronic alerts in the outpatient setting, ${ }^{13,14}$ the authors of this study hypothesized similar improvements should result from inpatient use of these interventions. Therefore, a pop-up alert specific to the adult psychiatry service was created in the University of Michigan (UM) Hospital CPOE system and implemented on 3 December 2008. The alert prompted clinicians prescribing scheduled SGAs to order fasting lipid and glucose levels for metabolic monitoring (Fig. 1). We evaluated the effectiveness of this metabolic monitoring alert on the successful collection of fasting lipid and blood glucose levels for adult psychiatry inpatients treated with SGAs by tracking the rates of monitoring of metabolic laboratory parameters before and after implementation.

\section{METHODS}

\section{Study site}

Our Adult Psychiatric Unit is a 22-bed general psychiatric unit with approximately 700 admissions per year and a 9-day average length of stay. The Adult Psychiatric Unit accepts both voluntary and involuntary patients, and the most common diagnoses include major depression (30\%), personality disorders (30\%), schizophrenia and schizoaffective disorder (15-20\%), bipolar I disorder (15\%) and substance abuse (15\%). Of note, 15-20\% of patients seen in the Adult Psychiatric Unit have comorbid medical conditions, some of which require management by other medical teams.

\section{Popup alert development and use}

Verbal reminders to prescribers from a clinical psychiatric pharmacist regarding the need for metabolic monitoring with SGAs had been ongoing. The clinical psychiatric pharmacist partnered with information technology to create a new pop-up alert following implementation of CPOE to facilitate systematic reminders (Fig. 1). Input from 'champion' psychiatrists was obtained to ensure adequate buy-in. The main objectives of the CPOE pop-up alert were to remind prescribers entering an SGA order to assess the need for metabolic monitoring, and to facilitate ordering of appropriate labs directly via the pop-up window. As the collection of patient weights and blood pressure measurements was considered standard of care, these parameters were not included in the pop-up alert. In addition, the collection of information regarding family history and waist circumference was not prompted by the pop-up alert and was not assessed as part of this study. To minimize impact on prescribers, the alert could be bypassed without reviewing previous laboratory results or submitting new laboratory orders. Whether or not the alert was bypassed, it would reappear with every subsequent scheduled SGA order, including dosage changes. The alert window populated with patient-specific glucose and lipid values obtained at any UMHS site within the previous 12 weeks. If values for glucose and/or lipid results were not displayed, the prescriber was to infer no recent values were collected. The alert also provided a citation ${ }^{2}$ where more information regarding SGA-related metabolic monitoring recommendations was available.

\section{Study design}

The impact of the UM CPOE pop-up alert was evaluated using an uncontrolled pre-post design: a retrospective chart review was performed in which patient demographic information as well as SGA drug and laboratory order data were extracted
Fig. 1. Screenshot of computerized physician order entry (CPOE) pop-up alert.

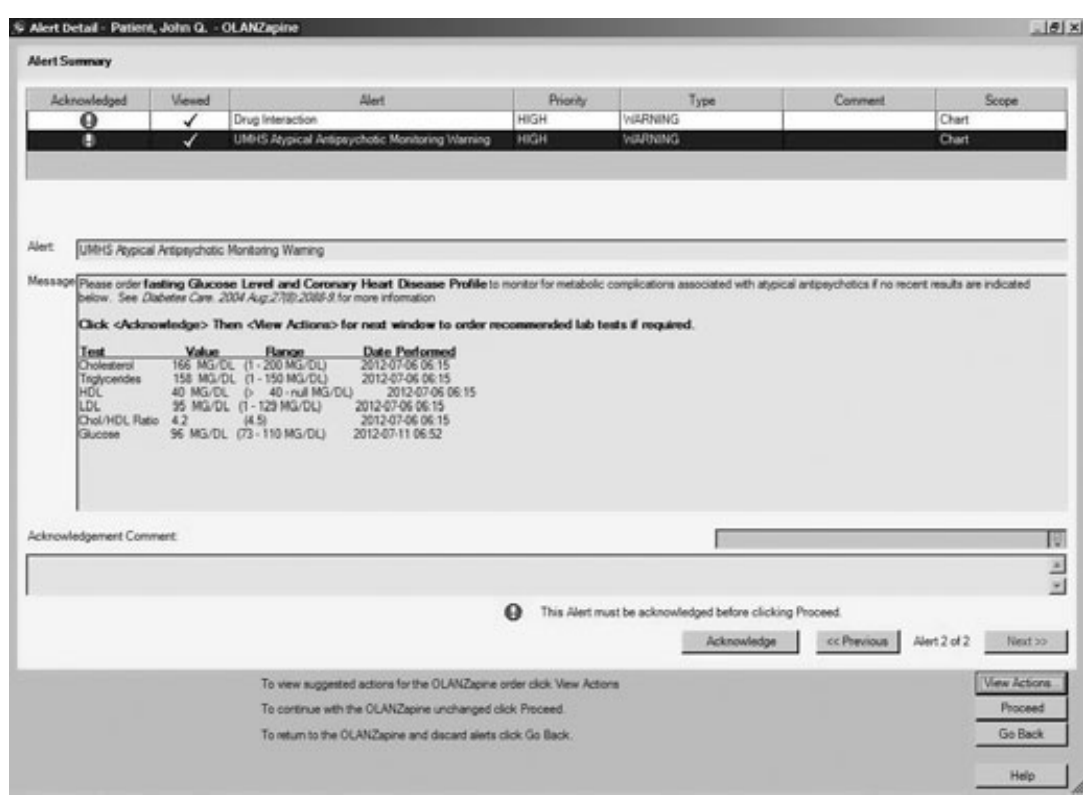


from the CPOE database for adult psychiatric patients admitted between 2 June 2008 and 3 June 2009. The number of patients on SGA therapy admitted between 2 June 2008 and 2 December 2008 for which fasting blood glucose levels and fasting lipid panels were available were compared to those patients admitted between 3 December 2008 and 3 June 2009. This study was approved by the UM Institutional Review Board.

\section{Patient population}

Patients were included if they were at least 18 years of age, and prescribed a scheduled SGA during the study period.

Only data from the first admission were included in the analysis. Patients were excluded from the study if they received only a one-time dose of an SGA $(N=6)$ or had SGA orders entered both before and after implementation of the alert $(N=6)$. Patients receiving SGAs on an as-needed basis only were also excluded, as the monitoring alert was not programmed to activate for 'p.r.n.' drug orders.

\section{Data collection}

Data extracted included patient demographics, admission date, SGA drug order information, and the date and time at which metabolic labs were ordered and collected.

\section{Outcomes}

The primary outcome measure of this study was the number of patients on SGA therapy for which fasting blood glucose levels and lipid panels were either ordered or available for review during the 6-month periods before and after alert implementation. Levels were considered fasting if drawn prior to 09:00, the start of breakfast service on the inpatient psychiatry unit. Patients were considered to have the appropriate metabolic labs available if they were collected within 12 weeks prior to the SGA order entry, or after the SGA was ordered but prior to discharge from the psychiatry unit.

Secondary outcomes included average time between entering an SGA order and subsequent metabolic monitoring laboratory orders, and the percentage of patients with the necessary metabolic monitoring labs ordered each month for 6 months after the pop-up alert was implemented. The latter was evaluated to determine if ordering frequency was sustained consistently over time following alert implementation.

\section{Statistical analysis}

Chi-squared and Student's $t$-tests were performed to compare pre-alert and post-alert patient demographics and rates of metabolic monitoring. A $P$-value of $<0.05$ was considered significant. Time trends in rates of laboratory ordering over the 6 months after implementation of the alert were examined using the Cochran-Armitage Trend test.

\section{RESULTS AND DISCUSSION}

\section{Patient demographics}

The pre- and post-alert groups included 171 and 157 patients, respectively. No significant differences existed between the two groups with respect to age, length of stay, sex, race or comor- bidities (Appendix). Additionally, there was no significant difference between groups when comparing the initial SGA agent prescribed. Twenty to twenty-three per cent of all pre- and post-alert patients started on agents deemed to be either high risk (clozapine or olanzapine) or low risk (aripiprazole or ziprasidone) for the development of SGA-related metabolic side effects. All other patients were prescribed quetiapine, risperidone or paliperidone.

\section{Rates of metabolic monitoring}

A statistically significant difference in availability of metabolic monitoring data was noted between patients admitted before and after implementation of the alert (Table 1). Patients in the post-alert group were more likely to have (i) a glucose or lipid level (fasting or random); (ii) a fasting glucose or lipid level; (iii) both a fasting glucose and fasting lipid level $(P \leq 0.001$ for each). Significantly more glucose and lipid labs post-alert were ordered either at the same time or within $24 \mathrm{~h}$ of the SGA (Table 1). This difference remained significant when narrowed to include only those labs ultimately drawn as fasting levels.

Time trend analysis indicated rates of laboratory monitoring did not decline over the 6-month study period post-implementation.

\section{Discussion}

Implementation of a CPOE pop-up alert reminding prescribers to order metabolic labs for patients on SGAs resulted in significant increases in rates of laboratory monitoring. The change in glucose level availability (fasting or random) between the pre- and postalert groups was less drastic than that of lipid panel availability ( $8 \%$ increase vs. $46 \%$ increase). Indeed, for general monitoring purposes, most patients provide a blood panel at some point during admission that includes a glucose measurement.

The significant increase in number of patients with a fasting glucose level post-alert may be related to the corresponding increase in ordering of stand-alone glucose levels facilitated by the alert. Whereas basic and comprehensive panels are frequently drawn throughout the day, glucose labs drawn separately are typically drawn as part of a morning collection process. There is a higher likelihood of individual glucose being drawn in a fasting state, especially when prescribers either provide special instructions within the order requesting that the level be collected fasting, or enter the order for time-critical collection in the morning.

Lipid panels are not included in routine monitoring blood work and are therefore ordered only when deemed necessary for specific patients. The increase from 30\% (pre-alert) to $70 \%$ (post-alert) of patients having lipid panels suggests the alert had a larger impact on the collection of samples for lipids. Rates of obtaining lipid panels in a fasting state, whereas increased post-alert, are still an area for improvement, as they remain below our goal of $100 \%$. Owing to technical constraints related to the CPOE system, we have not yet been able to pre-set the laboratory orders included in the alert box as 'fasting draws'; we hope additional features will be added in the future that allow for improvements in the appropriateness of both glucose and lipid panel collection. The availability of glucose and lipid levels together provides prescribers with a clearer picture of the potential consequences of SGA therapy. As shown in Table 1, significantly more patients admitted post-alert had both glucose 
Table 1. Patients for which a glucose level, lipid panel or both was/were available for metabolic monitoring purposes

\begin{tabular}{|c|c|c|c|}
\hline & Pre-alert $(n=171)$ & Post-alert $(n=157)$ & $P$-value \\
\hline \multicolumn{4}{|l|}{ Glucose levels } \\
\hline Patients with glucose level available & $158(92 \cdot 4)$ & $157(100)$ & 0.001 \\
\hline Subset drawn fasting ${ }^{\mathrm{a}}$ & $80(50 \cdot 6)$ & $110(70 \cdot 0)$ & $<0 \cdot 0001$ \\
\hline Patients with fasting ${ }^{a}$ glucose level available (overall) & $80(46 \cdot 8)$ & $110(70 \cdot 0)$ & $<0.0001$ \\
\hline Level ordered with SGA & $9(5 \cdot 7)$ & $31(19 \cdot 7)$ & $<0 \cdot 0001$ \\
\hline Subset drawn fasting & $4(2 \cdot 5)$ & $23(14 \cdot 6)$ & $<0 \cdot 0001$ \\
\hline Level ordered within $24 \mathrm{~h}$ of SGA & $31(19 \cdot 6)$ & $63(40 \cdot 1)$ & $<0 \cdot 0001$ \\
\hline Subset drawn fasting & $12(7 \cdot 6)$ & $46(29 \cdot 3)$ & $<0 \cdot 0001$ \\
\hline \multicolumn{4}{|l|}{ Lipid panels } \\
\hline Patients with lipid panel available & $49(28 \cdot 7)$ & $117(74 \cdot 5)$ & $<0 \cdot 0001$ \\
\hline Subset drawn fasting ${ }^{a}$ & $32(64 \cdot 0)$ & $94(81 \cdot 0)$ & 0.031 \\
\hline Patients with fasting ${ }^{a}$ lipid panel available (overall) & $32(18 \cdot 7)$ & $94(59 \cdot 9)$ & $<0 \cdot 0001$ \\
\hline Panel ordered with SGA & $4(8 \cdot 2)$ & $38(32 \cdot 5)$ & 0.002 \\
\hline Subset drawn fasting ${ }^{a}$ & $3(6 \cdot 1)$ & $30(25 \cdot 6)$ & $0 \cdot 008$ \\
\hline Panel ordered within $24 \mathrm{~h}$ of SGA & $12(24 \cdot 5)$ & $57(48 \cdot 7)$ & 0.007 \\
\hline Subset drawn fasting ${ }^{\mathrm{a}}$ & $9(18 \cdot 3)$ & $48(41 \cdot 0)$ & 0.009 \\
\hline \multicolumn{4}{|l|}{ Both (glucose and lipid) } \\
\hline Patients with glucose level and lipid panel available & $47(27 \cdot 5)$ & $117(74 \cdot 5)$ & $<0 \cdot 0001$ \\
\hline Fasting a glucose level and lipid panel available & $22(12 \cdot 9)$ & $75(47 \cdot 8)$ & $<0.0001$ \\
\hline
\end{tabular}

SGA, second-generation antipsychotic.

Data presented as $n(\%)$; Labs were included if they were drawn within the previous 12 weeks of SGA order entry or during study admission prior to discharge from inpatient psychiatry unit.

${ }^{\text {a } F a s t i n g ~ l e v e l s ~ w e r e ~ d r a w n ~ b e f o r e ~ 09: 00 ~(s t a r t ~ o f ~ b r e a k f a s t ~ s e r v i c e) . ~}$

and lipid levels available for review, even when considering only those levels drawn in a fasting state. This suggests the use of a pop-up alert can improve appropriate attainment of baseline glucose and lipid values in psychiatry inpatients on SGAs.

Rates of fasting laboratory monitoring remain low overall with $<50 \%$ of patients having both a fasting glucose and lipid measurement. These results demonstrate the need for additional strategies to further increase metabolic monitoring rates. These might include provision of educational in-services for prescribers or modification of the alert to prevent clinicians from bypassing it without ordering the appropriate labs.

One of the possible confounding factors of this study was the involvement of a clinical psychiatric pharmacist on the inpatient psychiatry unit throughout the study period. The psychiatric pharmacist impacts metabolic monitoring of patients on SGA therapy by reminding clinicians to order labs when appropriate. In an attempt to tease out the effect of the alert itself on the ordering and collection of metabolic monitoring labs, the time between the ordering of the SGA and that of the nearest glucose level and lipid panel was calculated. More glucose and lipid labs post-alert were ordered at the same time as the SGA, with nearly $20 \%$ of glucose levels and over $30 \%$ of lipid panels having been ordered at the same time as the SGA in patients admitted after alert implementation. This suggests that the alert itself may directly influence ordering of the necessary metabolic monitoring labs by facilitating the process and allowing prescribers to order the necessary labs directly within the alert box. Additionally, significantly more metabolic labs were ordered within $24 \mathrm{~h}$ of the SGA, suggesting that even when labs are not ordered directly within the alert box itself, the alert is able to successfully remind clinicians to order them separately shortly after submitting the SGA order.
Our study has several limitations. The retrospective design is less rigorous than a controlled, prospective design, which would be difficult to implement on a single unit. Pre- and postalert subject groups appeared comparable based on demographic and treatment parameters, but short of a randomized design, other group differences might have been present that we could not control. Additionally, our study included only laboratory results collected at a UMHS affiliated facility; if labs were drawn at another facility, we would have underestimated the presence of metabolic monitoring data. However, such an underestimation would be present throughout the study period and would therefore have negligible effects on our pre- and post-alert comparisons.

The option for prescribers to bypass the alert without ordering labs likely accounts for the proportion of post-alert patients lacking the proper monitoring. A concern with the alert is that of desensitization ('alert-fatigue') owing to the fact that the alert is triggered for all scheduled SGA orders, even when multiple orders are entered on the same patient within days or even minutes of each other. ${ }^{15}$ The stable trend of laboratory ordering over the 6 months post-alert suggests this was not a major factor. The alert is not currently programmed to appear for as-needed ('p.r.n.') orders, so metabolic monitoring may be overlooked in some patients that are taking SGA agents regularly but as part of a p.r.n. order (e.g. 'daily p.r.n.' or 'BID p.r.n.'). In addition, the alert currently emphasizes two of the six monitoring parameters recommended in the 2004 Consensus Statement, such that even proper collection of these labs may not provide a complete picture of the metabolic status of patients on SGAs.

Owing to limited lengths of stay in an inpatient setting, follow-up monitoring was not addressed in this study. The 
authors feel it is important to emphasize adequate baseline monitoring in inpatient settings, as hospital admissions frequently lead to medication changes and dose adjustment. Clearly communicated baseline assessments to outpatient providers are likely to enhance decision-making at outpatient visits. Whereas we recognize that improved monitoring alone does not improve health outcomes, it serves as an imperative first step to identifying the problem and working collaboratively with patients on SGA therapy to reduce their health risks.

Results of this study may assist other medical institutions in implementing programs to improve metabolic monitoring and patient care by encouraging and facilitating the collection of appropriate lipid and glucose measurements at baseline.

\section{WHAT IS NEW AND CONCLUSIONS}

Implementation and use of an electronic metabolic monitoring pop-up alert significantly improved collection of baseline fasting blood glucose and lipid levels for psychiatry inpatients treated with SGAs. Monitoring rates for these parameters overall, however, remain suboptimal, suggesting a need for the use of supplementary strategies to further improve metabolic monitoring for inpatients taking these medications. Further studies are needed to evaluate the impact of this type of electronic pop-up alert on the clinical decision-making process and patient health outcomes.

\section{CONFLICT OF INTEREST}

The authors declare no conflicts of interest.

\section{REFERENCES}

1. Sernyak MJ. Implementation of monitoring and management guidelines for second-generation antipsychotics. J Clin Psychiatry, 2007;68(Suppl. 4):14-18.

2. American Diabetes Association, American Psychiatric Association, American Association of Clinical Endocrinologists, North American Association for the Study of Obesity. Consensus development conference on antipsychotic drugs and obesity and diabetes. J Clin Psychiatry, 2004;65:267-272.

3. Straker D, Correll C, Kramer-Ginsberg E et al. Cost-effective screening for the metabolic syndrome in patients treated with second-generation antipsychotic medications. Am J Psychiatry, 2005;162:1217-1220.

4. Reeves R, Kaldany H, Lieberman H, Vyas R. Creation of a metabolic monitoring program for second-generation (atypical) antipsychotics. J Correct Health Care, 2009;15:292-301.

5. Newcomer JW. Antipsychotic medications: metabolic and cardiovascular risk. J Clin Psychiatry, 2007;68(Suppl. 4):8-12.

6. Chwastiak LA, Rosenheck RA, Kazis LE. Association of psychiatric illness and obesity, physical inactivity, and smoking among a national sample of veterans. Psychosomatics, 2011;52:230-236.

7. Ganguli R, Strassnig M. Prevention of metabolic syndrome in serious mental illness. Psychiatr Clin North Am, 2011;34:109125

8. Haupt DW, Rosenblatt LC, Kim E, Baker RA, Whitehead R, Newcomer JW. Prevalence and predictors of lipid and glucose monitoring in commercially insured patients treated with second-generation antipsychotic agents. Am J Psychiatry, 2009;166:345-353.

9. Morrato EH, Newcomer JW, Allen RR, Valuck RJ. Prevalence of baseline serum glucose and lipid testing in users of second-generation antipsychotic drugs: a retrospective, population-based study of Medicaid claims data. I Clin Psychiatry, 2008;69:316-322.

10. Morrato EH, Newcomer JW, Kamat $\mathrm{S}$, Baser O, Harnett J, Cuffel B. Metabolic screening after the American Diabetes Association's consensus statement on antipsychotic drugs and diabetes. Diabetes Care, 2009;32:1037-1042.
11. Morrato EH, Druss B, Hartung DM, Valuck RJ, Allen R, Campagna E, Newcomer JW. Metabolic testing rates in 3 state Medicaid programs after FDA warnings and ADA/APA recommendations for secondgeneration antipsychotic drugs. Arch Gen Psychiatry, 2010;67:17-24.

12. Schneiderhan ME, Batscha CL, Rosen C. Assessment of a point-of-care metabolic risk screening program in outpatients receiving antipsychotic agents. Pharmacotherapy, 2009;29:975-987.

13. Feldstein AC, Smith DH, Perrin N et al. Improved therapeutic monitoring with several interventions: a randomized trial. Arch Intern Med, 2006;166:1848-1854.

14. Were MC, Shen C, Tierney WM et al. Evaluation of computer-generated reminders to improve CD4 laboratory monitoring in sub-Saharan Africa: a prospective comparative study. I Am Med Inform Assoc, 2011;18:150-155.

15. Lo HG, Matheny ME, Seger DL, Bates DW, Gandhi TK. Impact of non-interruptive medication laboratory monitoring alerts in ambulatory care. J Am Med Inform Assoc, 2009;16:66-71. 
Appendix: Patient Demographics of Pre- and Post-Alert Populations

\begin{tabular}{|c|c|c|c|}
\hline & Before alert $(n=171)$ & After alert $(n=157)$ & $P$-value \\
\hline Average age (years $\pm \mathrm{SD}$ ) & $43 \pm 16$ & $42 \pm 15$ & $0 \cdot 423$ \\
\hline Length of stay (days \pm SD) & $12 \cdot 5 \pm 13 \cdot 2$ & $13 \cdot 4 \pm 13 \cdot 5$ & 0.549 \\
\hline \multicolumn{4}{|l|}{ Comorbidities } \\
\hline Diabetes & $16(9 \cdot 4)$ & $20(12 \cdot 7)$ & $0 \cdot 423$ \\
\hline Hyperlipidaemia & $19(11 \cdot 1)$ & $20(12 \cdot 7)$ & 0.776 \\
\hline Coronary artery disease/cardiovascular disease & $1(0 \cdot 6)$ & $4(2 \cdot 5)$ & 0.318 \\
\hline BMI $>25$ or diagnosis of 'Obesity' & $12(7 \cdot 0)$ & $9(5 \cdot 7)$ & 0.803 \\
\hline Patients $w / \geq 1$ of the above & $36(21 \cdot 1)$ & $41(26 \cdot 1)$ & 0.342 \\
\hline \multicolumn{4}{|l|}{ Race } \\
\hline Caucasian & $136(79 \cdot 5)$ & $126(80 \cdot 3)$ & 0.980 \\
\hline Black/African American & $21(12 \cdot 3)$ & $16(10 \cdot 2)$ & $0 \cdot 672$ \\
\hline Hispanic & $2(1 \cdot 2)$ & $3(1.9)$ & 0.923 \\
\hline Native American & $1(0 \cdot 6)$ & $0(-)$ & 0.966 \\
\hline $\mathrm{Bi} /$ Multi-racial & $3(1 \cdot 8)$ & $1(0 \cdot 6)$ & $0 \cdot 676$ \\
\hline Asian & $3(1 \cdot 8)$ & $3(1.9)$ & 0.759 \\
\hline Middle Eastern & $1(0 \cdot 6)$ & $2(1 \cdot 3)$ & 0.941 \\
\hline Unknown/Other & $1(0 \cdot 6)$ & $2(1 \cdot 3)$ & 0.941 \\
\hline Not listed & $3(1 \cdot 8)$ & $4(2 \cdot 6)$ & 0.909 \\
\hline \multicolumn{4}{|l|}{ Sex } \\
\hline Male & $77(45)$ & $77(49)$ & 0.537 \\
\hline Female & $94(55)$ & $80(51)$ & 0.537 \\
\hline \multicolumn{4}{|l|}{ Discharge diagnoses $^{\mathrm{a}}$} \\
\hline Schizophrenia/psychotic disorders & $62(36 \cdot 3)$ & $44(28 \cdot 0)$ & $0 \cdot 140$ \\
\hline Depressive disorders & $43(25 \cdot 1)$ & $47(29 \cdot 9)$ & 0.434 \\
\hline Bipolar disorders & $35(20 \cdot 5)$ & $39(24 \cdot 8)$ & 0.415 \\
\hline Mood disorder NOS & $9(5 \cdot 3)$ & $11(7 \cdot 0)$ & 0.669 \\
\hline Personality disorders & $6(3.5)$ & $4(2 \cdot 5)$ & 0.854 \\
\hline Dementia & $2(1 \cdot 2)$ & $2(1 \cdot 3)$ & 0.676 \\
\hline Anxiety disorders & $2(1 \cdot 2)$ & $6(3 \cdot 8)$ & $0 \cdot 231$ \\
\hline Substance-related disorder & $5(2 \cdot 9)$ & $0(-)$ & 0.088 \\
\hline Adjustment disorder & $4(2 \cdot 3)$ & $1(0 \cdot 6)$ & $0 \cdot 420$ \\
\hline Other ${ }^{\mathrm{b}}$ & $3(1 \cdot 8)$ & $2(1 \cdot 3)$ & 0.966 \\
\hline \multicolumn{4}{|l|}{ SGA agent ordered ${ }^{c}$} \\
\hline Clozapine/Olanzapine (high risk) & $38(22 \cdot 2)$ & $35(22 \cdot 3)$ & 0.906 \\
\hline Aripiprazole/Ziprasidone (low risk) & $36(21 \cdot 0)$ & $37(23 \cdot 6)$ & $1 \cdot 000$ \\
\hline
\end{tabular}

SD, standard deviation; SGA, second-generation antipsychotic.

Data presented as $n(\%)$ unless otherwise specified.

${ }^{\mathrm{a}}$ Groupings based on DSM-IV.

${ }^{\mathrm{b}}$ Somatoform disorder, autistic disorder, impulse control disorder, delirium, mood disorder (gen med condition).

'Only first agent ordered; changes in therapy may have occurred during the hospital stay. 